\title{
Study on A New Type Plunge Pool of High Arch Dam
}

\author{
Wang Yingkui ${ }^{1,}$ a, Zhu $\mathrm{Jia}^{2, \mathrm{~b}}$ and Jiang Chunbo ${ }^{3, \mathrm{c}}$ \\ ${ }^{1}$ Changjiang Institute of Survey, Planning, Design and Research, Wuhan Hubei 430010, China \\ ${ }^{2}$ Dongfeng Peugeot Citroen Automobile Company Ltd, Wuhan Hubei 430056, China \\ ${ }^{3}$ State key laboratory of Hydroscience and Engineering, Tsinghua University, Beijing 100084 , \\ China
}

âwangyingkui@cjwsjy.com.cn, ${ }^{b}$ zj_alice@163.com, ${ }^{c}$ jcb@mail.tsinghua.edu.cn

Keywords:High arch dam. Hydraulic model experiment, Energy dissipation, Newtype plunge pool. Abstract. Lots of built or building dams, especially the high arch dam, located in the Southwest China with abundant hydro-power resources. Most of these dams have the characteristics of "High head, Large discharge and Narrow river valley", therefore, the security researches of energy dissipation were always the focus in these hydro-projects. Statistically, the trajectory type energy dissipation is the most widely used in the built high arch dams at home and abroad, which have simple engineer structure, short construction period and the adaptability advantage on the changing discharge and Tail water depth. And the water plunge poor were always set downstream the dam body with large discharge and large power. However, the widely used protected plunge poor need large investment with the disadvantage of complicated operation and maintenance. Along with the construction of concrete high arch dam in the Southwest China, the river overburden and water cushion were deep in dam site, which is becoming a new characteristic of these hydro-projects. Accordingly, the deep water cushion can be used for the energy dissipation design, such as the "Reventment-Protected and Non-Bottom-Protected Plunge Pool", which has the advantage of more simplified project design and more economy investment.

\section{Introduction}

Lots of built or building dams, especially the high arch dam, located in the Southwest China with abundant hydro-power resources. Most of these dams have the characteristics of "High head, Large discharge and Narrow river valley", and there are lots of difficulties in form choice, entire layout and structure design, which can affect the energy dissipation. The security of energy dissipation not only can influent the project investment and benefit, and also have direct influence on the safety operation of the hydro-project. So the research were always the focus in these hydro-projects, especially in high arch dam projects ${ }^{[1]}$.

In the built high arch dams, the trajectory type energy dissipation is the most widely used in dam body, and the water plunge poor were set downstream the dam body for the project safety. However, the widely used protected plunge poor need large investment with the disadvantage of complicated operation and maintenance ${ }^{[2]}$. Along with the construction of concrete high arch dam in the Southwest China, the river overburden and water cushion were deep in dam site, which is becoming a new characteristic of these hydro-projects. Accordingly, the deep water cushion can be used for the energy dissipation design, which can simple the concrete-protected design and save the investments.

The energy dissipation mechanism of plunge pool is always studied based on the submerged impinging jet theory, including the water pattern, turbulent diffusion rule, flow velocity and impact pressure distribution rule, turbulent fluctuation process in the plunge pool. It's more complicated for the energy dissipation using the water cushion in nature river bed and there is no representative research work. In this article, based on the submerged impinging jet theory, the energy dissipation mechanism in water plunge poor was discussed in this article. And then the plunge poor patterns were summarized in combined with the engineering practice. A new type - "Reventment-Protected and Non-Bottom-Protected Plunge Pool" was proposed. 


\section{Theoretical considerations}

The flood discharge into the plunge pool is always considered as the submerged impinging jet flow, and that's why the research is studied based on the submerged impinging jet theory.

Turbulent impinging jet is the most popular jet flow, so the mechanisms of inside energy transfer, momentum transport, fluid entrainment, mixing and diffusion are closely related with the vortex structure caused by the jet velocity gradient. Early in 1953, Batchelor ${ }^{[3]}$ studied the turbulent characteristics of vortex structure in the jet flow. There are lots of systemical theory achievements in the research of free turbulent jet flow and also many experimental research work of submerged impinging jet flow.

There will be slanting free-impinging jet flow when the water cushion is shallow. The discharge flow is totally exposed in the air and flush into the bottom of the plunge pool. There will be free hydraulic jump next to the impinging area. This flow pattern is usually appeared in Multi-waterfall plunge pool with small water head. When the water head is higher, the impact pressure in the bottom will be too large, the flow pattern in plunge pool is turbulent and unsteady, the bottom is easy to be cavitation, therefore, this flow pattern should be avoid in the energy dissipation of high dam ${ }^{[4,5]}$.

When the water cushion is deep enough, it will protect the plunge pool bottom from the direct impinging flush. The jet impinging section is under water with large submerged, so there will be submerged impinging jet flow and submerged hydraulic jump in the plunge pool. The energy dissipation in the plunge pools of high dam are mostly belongs to this flow pattern. There are 3 flow regions: submerged jet flow area, impinging area and bottom jet flow area.

In the engineering practice, the flood discharge into the plunge pool of high arch dam will become the submerged impinging jet flow. The researchers has deeply studied the flow pattern, velocity distribution, bottom impact pressure, erosion distribution and structure vibration of the plunge pool and so on ${ }^{[6,7]}$. Many compared analysis were proposed based on the model experimental and original observation $^{[8,9]}$, which can provide the theory support for the energy dissipation design of high arch dams.

\section{Mechanism of energy dissipation}

The slanting submerged impinging jet flow in plunge pool can be considered as the mixing of main jet flow area and vortex structure area, and there is a strong turbulent shear zone between the main jet flow area and vortex structure area, where the mechanical energy can be reduced by the turbulent shear and diffuse, so it's the major region of energy dissipation. The mechanical energy dissipated is partially transferred to form the turbulent vortex, maintaining the turbulent fluctuation and dissipation; partially transferred to macroscopic vortex area, providing the rotation energy needs; and there are little part of energy dissipated by the shear effect in the transformation process. Liu Peiqing ${ }^{[10]}$, Head ${ }^{[11]}$, Acarlar et $\mathrm{al}^{[12]}$ studied the macroscopic vortex structure in plunge pool, proposed the partition areas and studied the development process of vortex structures in main jet flow area and bottom jet flow area. Xu Weilin ${ }^{[13]}$ studied the hydraulic characteristics of turbulent flow in plunge pool by numerical simulation, and carried out the research work of energy dissipation mechanism compared with the model experiment results.

Experimental results showed that: the mechanical energy of flood discharge reduced gradually along the diffusion process, most of the energy dissipated is transferred to turbulent fluctuation and others dissipated by the shear effect. And the simulation results showed that, the energy dissipation rate is $70.43 \%$ in the submerged jet flow area and $20.79 \%$ in the submerged hydraulic jump area. 


\section{New Type of plunge pool}

Totally protected plunge pool

There are large discharge and flood discharge capacity in the built or building high arch dams with manual plunge pool for energy dissipation. Almost all the plunge pool is total protected, which has two most common structure type: flat-bottom plunge pool and invert-arch plunge fool.

The flat-bottom plunge pool has concrete floor laid in the middle of river bed, which should meet the stability principle of gravity, and the concrete floor is mostly very thick to resist the huge buoyancy with the measures of pump drainage and anchorage et al.

The invert-arch plunge pool is designed according with the river nature shape, which is arch style concrete bottom instead of flat-bottom. The stability's controlling conditions of invert-arch plunge pool is based on the arch ring stability, and the huge buoyancy is resisted by the arch effect, which can increase the structure's security.

Partially protected plunge pool

Partially protected plunge pool can use the deep water cushion for energy dissipation, and only part of the bottom or bank was protected by the concrete plate. Such as "Reventment-Protected and Non-Bottom-Protected", "Bottom-Protected and Non- Reventment-Protected", "Partially Reventment-Protected" and "Partially Bottom-Protected" et al which has the conditions that nature rock is contacted with the discharge flood, as shown in Figure 1.

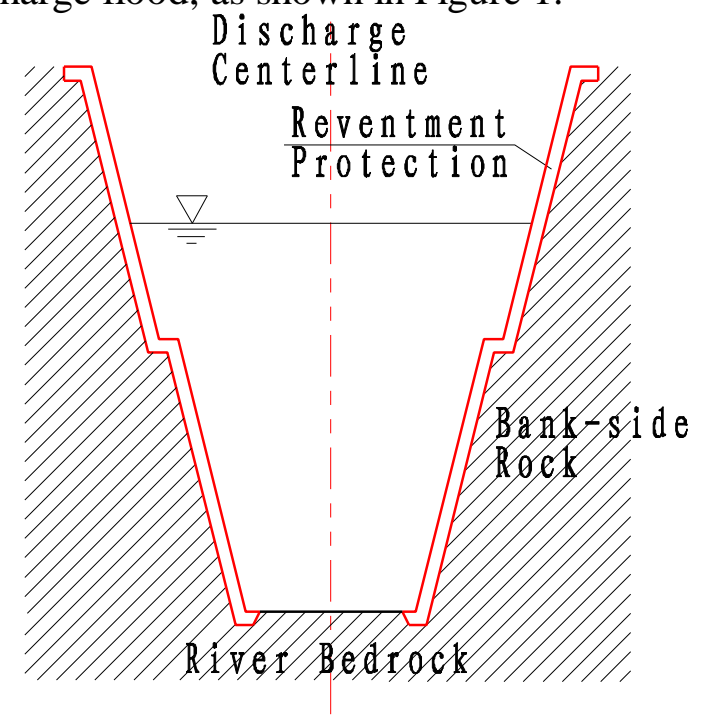

Figure 1 Sketch of Reventment-Protected and Non-Bottom-Protected plunge pool

The design of partially protected plunge pool is rare in the high arch dams built or building. However, it has obvious advantage in some special engineering conditions. There are some high arch dams in building with deep river overburden and water cushion, and the totally protected plunge poor need large investment, long construction period, complicated operation and maintenance. So it is possible to take the design of partially protected plunge pool with advantage of deep water cushion.

\section{Conclusions}

Due to the huge flood discharge capacity, the trajectory type energy dissipation is the most widely used in dam body with manual totally protect plunge pool. The submerged impinging jet flow area is the major region of energy dissipation, so the water cushion depth is a very important influence factor. When the water cushion is very deep in the high arch dam site, the deep water cushion can be considered for the energy dissipation, which can simple the concrete-protected design. Therefore, the design of partially protected plunge pool was proposed. 


\section{Acknowledgements}

This paper is supported by the CRSRI Open Research Program (Program SN : CKWV2014203/KY).

\section{References:}

[1] Zhou Jianping, Yang Zeyan, Chen Guanfu. Status and challenges of high dam constructions in China. Journal of Hydraulic Engineering, 2006, 37(12): 1433-1438;

[2] Liu Peiqing, Xu Weilin. Study on non-impact energy dissipation of ski-drop flow in plunge pool of high arch dam. Journal of Hydraulic Engineering, 2010, 41(7): 841-848;

[3] Batchelor G H. The theory of homogeneous turbulence. New York: Cambridge University Press, 1953.

[4] Yu Chanzhao. Turbulent Jet flow. Beijing: Higher Education Press, 1993;

[5] Liu Peiqing. Free turbulent jet flow theory. Beijing: Beihang University Press, 2008;

[6] Tian Zhong, Xu Weilin, Wang Wei, Liu Shanjun. Experimental study on impinging pressure caused by high velocity submerged jet. Journal of Hydraulic Engineering, 2005, 36(4): 401-404;

[7] Li Aihua, Liu Peiqing. Mechanism of disintegration of rocky riverbed under the action of fluctuating pressure due to impinging jet. Journal of Hydraulic Engineering, 2007, 38(11): 1324-1328;

[8] Han Xijun, Han Jibin, Chen Zibing. Enengy dissipation features \& hydraulic model test of Wudongde Hydropower Station. Hubei Water Power, 2007, 30:40-43;

[9] Zhang Chunman, Liu Peiqing, Liu Xianliang. The Impact Pressure Characteristic Research on Jet-f lows of High Arc Dams in Gorge Areas. Journal of Irrig at ion and Drainage, 2010, 29(2):109-111;

[10] Liu Peiqing, Li Futian. Large vortex structures and their characteristics of submerged impinging jet in plunge pool. Journal of Hydraulic Engineering, 2000, 1: 60-66;

[11] Head, M. R., Bandyopad hyay P., New aspects of turbulent boundary layer structure. J. Fluid Mech., 1981, 107:297-338.

[12] Acarlar M. S., Smith, C. R., A study of hairpin vortices in a laminar boundary layer, Part. II, hairpin vortices generated by fluid injection, J. Fluid Mech., 1987, 175:43-85.

[13] Xu Weilin, Liao Huashen, Yang Yongquan, Wu Chigong. Numerical simulation of 3-D turbulent flows of plunge pool and energy dissipation analysis. Journal of Hydrodynamics. 1996, 11(5): 561-569 